\title{
ALMA Observations of the Water Fountain Pre-planetary Nebula IRAS 16342-3814: High-velocity Bipolar Jets and an Expanding Torus
}

\author{
R. Sahai ${ }^{1}$, W. H. T. Vlemmings ${ }^{2}$, T. Gledhill ${ }^{3}$, C. Sánchez Contreras ${ }^{4}$, E. Lagadec ${ }^{5}$, \\ L-Å Nyman ${ }^{6,7}$, and G. Quintana-Lacaci ${ }^{8}$ \\ 1 Jet Propulsion Laboratory, MS 183-900, California Institute of Technology, Pasadena, CA 91109, USA; raghvendra.sahai@jpl.nasa.gov \\ ${ }^{2}$ Department of Earth and Space Sciences, Chalmers University of Technology, Onsala Space Observatory, SE-43992 Onsala, Sweden \\ ${ }^{3}$ Centre for Astrophysics Research, University of Hertfordshire, College Lane, Hatfield AL10 9AB, UK \\ ${ }^{4}$ Astrobiology Center (CSIC-INTA), ESAC Campus, E-28691 Villanueva de la Canada, Madrid, Spain \\ ${ }^{5}$ Université Côte d'Azur, Observatoire de la Côte d'Azur, CNRS, Lagrange, France \\ ${ }^{6}$ Joint ALMA Observatory (JAO), Alonso de Cordova 3107, Vitacura, Santiago de Chile, Chile \\ ${ }^{7}$ European Southern Observatory, Alonso de Cordova 3107, Vitacura, Santiago, Chile \\ ${ }^{8}$ Instituto de Ciencia de Materiales de Madrid, Sor Juana Ines de la Cruz, 3, Cantoblanco, E-28049, Madrid, Spain \\ Received 2016 November 4; revised 2016 December 15; accepted 2016 December 15; published 2017 January 20
}

\begin{abstract}
We have mapped ${ }^{12} \mathrm{CO} J=3-2$ and other molecular lines from the "water fountain" bipolar pre-planetary nebula (PPN) IRAS 16342-3814 with $\sim 0$ ". 35 resolution using Atacama Large Millimeter/submillimeter Array. We find (i) two very high-speed knotty, jet-like molecular outflows; (ii) a central high-density $\left(>\right.$ few $\times 10^{6} \mathrm{~cm}^{-3}$ ), expanding torus of diameter $1300 \mathrm{au}$; and (iii) the circumstellar envelope of the progenitor AGB, generated by a sudden, very large increase in the mass-loss rate to $>3.5 \times 10^{-4} M_{\odot} \mathrm{yr}^{-1}$ in the past $\sim 455$ years. Strong continuum emission at $0.89 \mathrm{~mm}$ from a central source $(690 \mathrm{mJy})$, if due to thermally emitting dust, implies a substantial mass $\left(0.017 M_{\odot}\right)$ of very large ( $\sim$ millimeter-sized) grains. The measured expansion ages of the above structural components imply that the torus (age $\sim 160$ years) and the younger high-velocity outflow (age $\sim 110$ years) were formed soon after the sharp increase in the AGB mass-loss rate. Assuming a binary model for the jets in IRAS 16342, the high momentum rate for the dominant jet-outflow in IRAS 16342 implies a high minimum accretion rate, ruling out standard Bondi-Hoyle-Lyttleton wind accretion and wind Roche-lobe overflow (RLOF) models with white-dwarf or main-sequence companions. Most likely, enhanced RLOF from the primary or accretion modes operating within common-envelope evolution are needed.
\end{abstract}

Key words: circumstellar matter - stars: AGB and post-AGB - stars: mass-loss - stars: winds, outflows techniques: interferometric

\section{Introduction}

High-resolution imaging surveys of planetary nebulae (PNe) and pre-planetary nebulae (PPNe) reveal a dazzling variety of bipolar and multipolar morphologies in these objects (e.g., Sahai et al. 2007, 2011; Siódmiak et al. 2008). In contrast, their progenitor AGB stars are typically embedded in roughly spherical gas-dust circumstellar envelopes (CSEs) resulting from dense, slow (typically $\sim 10-25 \mathrm{~km} \mathrm{~s}^{-1}$ ) stellar winds ejected with rates up to $10^{-4} M_{\odot} \mathrm{yr}^{-1}$ (e.g., Neri et al. 1998; Castro-Carrizo et al. 2010). Among various mechanisms that have been considered (Balick \& Frank 2002), hydrodynamic sculpting of a prior, spherically symmetric outflow by wandering and/or episodic jets from the inside out (Sahai \& Trauger 1998) is widely believed to be the primary mechanism that can produce the extreme aspherical shapes in $\mathrm{PNe}$ and PPNe. Support for this mechanism comes from an increasing number of detailed observational studies of specific PPNe (Cox et al. 2000; Sahai et al. 2006, 2013; Olofsson et al. 2015) and numerical simulations of jet-CSE interactions (e.g., Lee \& Sahai 2003; Balick et al. 2013).

The key to understanding the jet-sculpting (or alternative) shaping mechanisms lies in the study of the structure and kinematics of the youngest objects: the "water-fountains"class of young PPNe in which unusually fast radial $\mathrm{H}_{2} \mathrm{O}$ outflows with $V_{\exp } \gtrsim 55 \mathrm{~km} \mathrm{~s}^{-1}$ (Likkel \& Morris 1988; Likkel et al. 1992) show that the jet activity is extremely recent $(\lesssim 100$ years: Imai 2007).
IRAS 16342-3814 (hereafter IRAS 16342) is the best-studied and nearest $(\sim 2 \mathrm{kpc})$ water fountain PPN. Its morphology is well resolved with optical (HST) and near-infrared (Keck Adaptive Optics) imaging. Radio interferometry (VLA, VLBA) shows water masers spread over a range of radial velocities encompassing $270 \mathrm{~km} \mathrm{~s}^{-1}$ (Sahai et al. 1999, Setal99; Claussen et al. 2009). Single-dish millimeter-wave CO $J=2-1$ and 3-2 observations (He et al. 2008; Imai et al. 2012) reveal a massive, high-velocity molecular outflow. The radio and optical data on IRAS 16342 suggest nebular shaping in action, with a fast collimated outflow creating a pair of bubble-like cavities by plowing into a surrounding dense, more slowly expanding AGB CSE. The near-IR AO imaging reveals a remarkable corkscrew structure inscribed on the lobe walls-inferred to be a signature of a precessing jet (Sahai et al. 2005: Setal05). Shock-excited $\mathrm{H}_{2}$ emission is found in the lobes, presumably excited by the jet (Gledhill \& Forde 2012). The central star remains obscured behind a dark equatorial waist even in the mid-IR (12 $\mu \mathrm{m}$; Verhoelst et al. 2009).

Here, we report millimeter-wave molecular-line and continuum interferometric observations of IRAS 16342, using the Atacama Large Millimeter/submillimeter Array (ALMA). These data clearly reveal, for the first time, the dynamics of a pair of young, high-speed, bipolar collimated outflows, the presence of a high-density central torus, and a surrounding CSE resulting from an enormous increase in the mass-loss rate in the past few hundred years. 

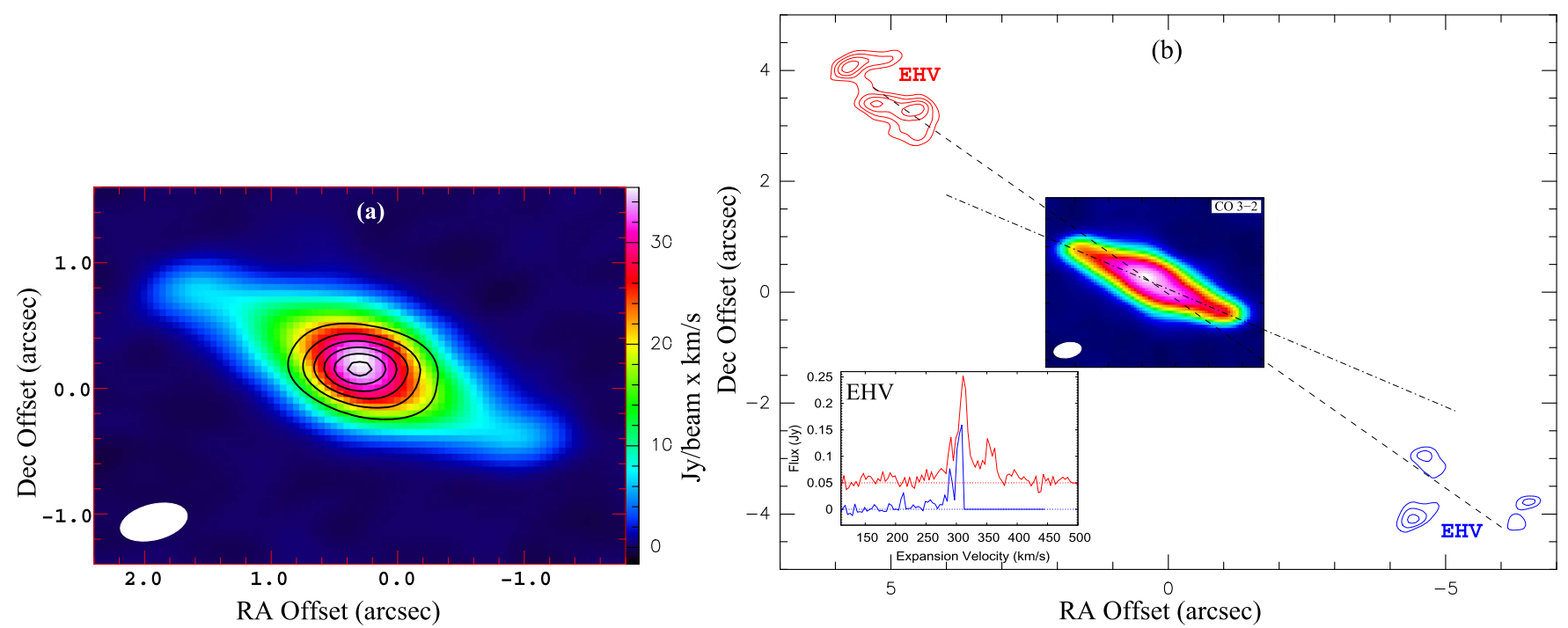

Figure 1. ALMA maps of the ${ }^{13} \mathrm{CO},{ }^{12} \mathrm{CO} J=3-2$, and $0.89 \mathrm{~mm}$ continuum emission from IRAS 16342 (white ellipses show the beam FWHM and orient). (a) ${ }^{13} \mathrm{CO}$ emission integrated over the HVO velocity range of $\pm 260 \mathrm{~km} \mathrm{~s}^{-1}$ centered at the systemic velocity. The continuum intensity is shown as contours, with the lowest level (step) equal to $20 \%(15 \%)$ of the peak, $0.25 \mathrm{Jy} \mathrm{beam}^{-1}$. The continuum peak is located at $(\mathrm{J} 2000)$ R.A. $=16: 37: 39.935$, decl. $=-38: 20: 17.15$, and the phase center (i.e., offset 0,0 ) at R.A. $=16: 37: 39.91$, decl. $=-38: 20: 17.3$. (b) Colorscale shows ${ }^{12} \mathrm{CO}$ emission integrated over the HVO velocity range. Contours show red- and blueshifted components of the EHVO covering emission at $\pm 310 \mathrm{~km} \mathrm{~s}^{-1}$ relative to the systemic velocity (the red emission is integrated over $34 \mathrm{~km} \mathrm{~s}^{-1}$, whereas the blue emission lies at the edge of the bandpass and is integrated over $24 \mathrm{~km} \mathrm{~s}^{-1}$ ). Inset shows the ${ }^{12} \mathrm{CO}$ spectra of the red- and blueshifted components of the EHVO, and the dashed (dash-dotted) line shows the axis of the EHVO (HVO).

\section{Observations}

The data were obtained on 2015 June 13 with 37 antennas of the ALMA $12 \mathrm{~m}$ array in band 7 . Using $35 \mathrm{~min}$ on the science target, the data cover four spectral windows (SPWs). Two $1.875 \mathrm{GHz}$-bandwidth SPWs were centered on $345.170 \mathrm{GHz}$ (SPW0) and $331.214 \mathrm{GHz}$ (SPW2) with 3840 channels each. Two additional continuum SPWs with $2 \mathrm{GHz}$ bandwidth with 128 channels were centered on $343.337 \mathrm{GHz}$ (SPW1) and $333.104 \mathrm{GHz}$ (SPW4). The baselines range from 21 to $783 \mathrm{~m}$ implying a maximum recoverable scale of $\sim 4^{\prime \prime}$. The data were calibrated using the ALMA pipeline using Ceres as flux calibrator and the quasars J1924-2914 ( 2.7 Jy) and J1636$4102(\sim 0.14 \mathrm{Jy})$ as bandpass and gain calibrator, respectively. Following the standard calibration, self-calibration was performed on the continuum emission of IRAS 16342. Images were created using a Briggs weighting scheme channel averaging to $4 \mathrm{~km} \mathrm{~s}^{-1}$ in continuum subtracted line SPWs. In the continuum SPWs, the effective channel width corresponds to $\sim 14 \mathrm{~km} \mathrm{~s}^{-1}$. Typical channel $\mathrm{rms}$ is $\sim 2.3 \mathrm{mJy}^{-1}$ beam $^{-1}$ in line-free $4 \mathrm{~km} \mathrm{~s}^{-1}$ channels.

\section{Results}

We have identified a number of lines in the 4 SPWs. In addition to the $J=3-2$ lines of ${ }^{12} \mathrm{CO}$ (SPW0) and ${ }^{13} \mathrm{CO}$ (SPW2), and $\mathrm{H}^{13} \mathrm{CN} J=4-3$ (SPW0) that are discussed here, we also find SO N, $J=8,8-7$, 7, (SPW0, SPW1), CS $J=7-6(\mathrm{SPW} 1),{ }^{29} \mathrm{SiO} J=8-7 \quad(v=0)(\mathrm{SPW} 1), \mathrm{SO}_{2} 4(3$, 1)-3(2, 2) (SPW3), and ${ }^{34} \mathrm{SON}, J=8,7-7,6$ (SPW3) (discussed in R. Sahai et al. 2017, in preparation).

\subsection{High-velocity Outflows}

The IRAS $16342{ }^{12} \mathrm{CO}$ and ${ }^{13} \mathrm{CO}$ moment-zero maps, generated by integrating over a velocity range $\pm 260 \mathrm{~km} \mathrm{~s}^{-1}$ relative to the systemic velocity $\left(V_{\mathrm{lsr}} \sim 45 \mathrm{~km} \mathrm{~s}^{-1}\right)$, show point-symmetric shapes (Figure 1(a)). The SW (NE) lobe is blueshifted (redshifted), and the nebula's long axis is oriented at $\mathrm{PA}=67^{\circ}$. The ${ }^{12} \mathrm{CO}$ image shows emission from an extreme high-velocity outflow (EHVO) located much further from the nebular tips at a (projected) radial velocity of $\sim \pm 310 \mathrm{~km} \mathrm{~s}^{-1}$ (Figure 1(b)), and the axis of this outflow is oriented at PA $\sim 55^{\circ}$. Since this emission has a high degree of symmetry relative to the center, in both position and velocity, and because it lies along an axis close to the nebular axis, we conclude that it is related to IRAS 16342, and not to random interstellar clouds in the Galactic plane. A comparison of the expansion velocity ranges of the blue- and redshifted EHVO components (Figure 1(b), inset) indicates that part of the former lies beyond the bandpass edge.

Position-velocity (PV) plots of ${ }^{12} \mathrm{CO} J=3-2$ and $\mathrm{H}^{13} \mathrm{CN}$ $J=4-3$ emission along the nebular axis generally show an S-shape, with small knots (Figure 2(a)). We have identified a total of five pairs of such knots $(B 1-B 5$ and $R 1-R 5)$. The emission from the central bipolar nebula extends over $-230<V_{\mathrm{lsr}}\left(\mathrm{km} \mathrm{s}^{-1}\right)<325$ in ${ }^{12} \mathrm{CO}$ (Figure 2(b)); the emission from ${ }^{13} \mathrm{CO} J=3-2$ and $\mathrm{H}^{13} \mathrm{CN} J=4-3$ is weaker and is detectable over a somewhat smaller range $\left(\sim 500 \mathrm{~km} \mathrm{~s}^{-1}\right)$.

The spiral structure seen in the PV plots most likely indicates emission from a precessing high-velocity bipolar outflow (HVO) that entrains material in the near and far bipolar lobe walls. Blobs $B 1$ and $R 1$ have the highest outflow velocities and the smallest spatial offset from the center and are thus moving along an axis close to the line-of-sight (los), implying an outflow speed of slightly more than $250 \mathrm{~km} \mathrm{~s}^{-1}$. If we assume that all blobs are moving radially with this same outflow speed, i.e., $250 \mathrm{~km} \mathrm{~s}^{-1}$, then we can derive the locations of blob pairs $B 2-R 2, B 3-R 3, B 4-R 4$, and $B 5-R 5$, along the los and their ages $(\sim 95,80,60,20$ years, respectively; Figure 3$)$. The $B 1-R 1$ pair's much lower brightness, compared to that of the other pairs, suggests that $B 1-R 1$ is the oldest pair, since we expect the blobs to cool and dissipate with time. Assuming that the interval between the ejections of $B 1-R 1$ and $B 2-R 2$ is similar to that between $B 2-R 2$ and $B 3-R 3$, the $B 1-R 1$ 


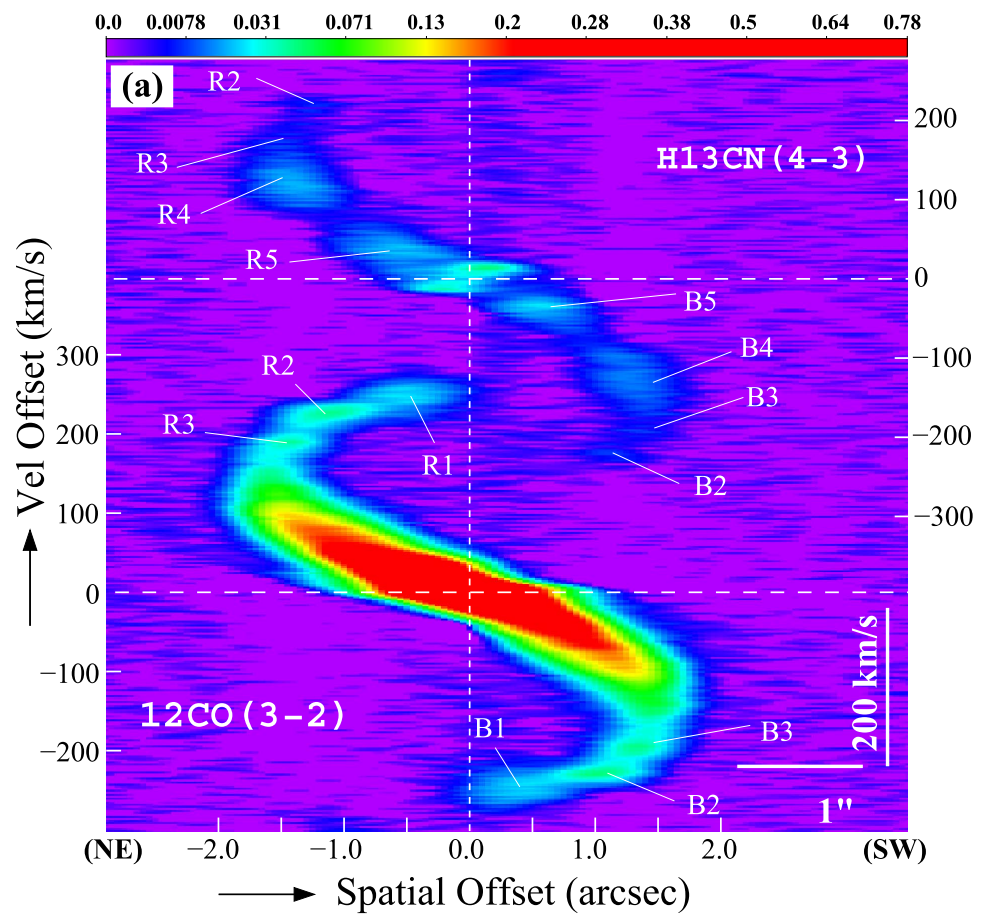

(b)

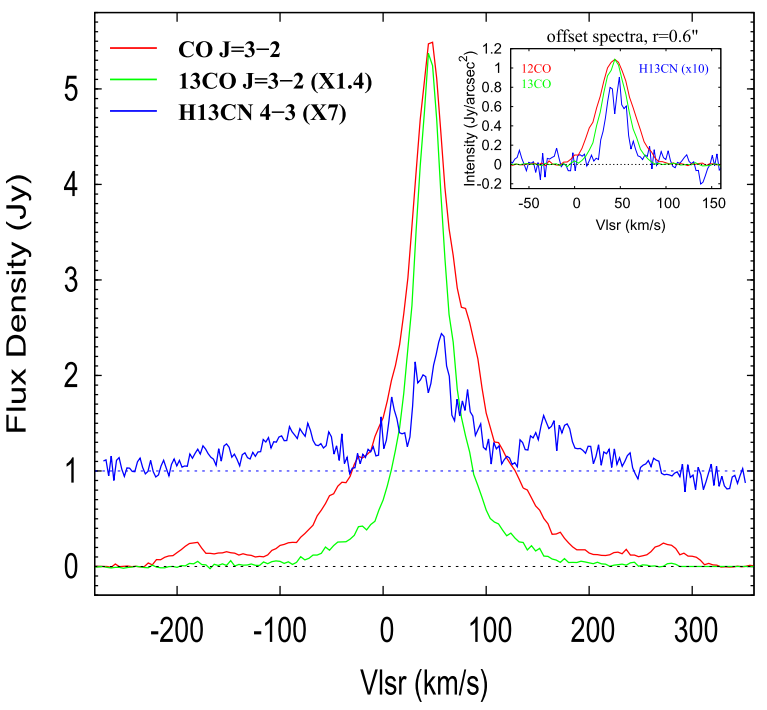

Figure 2. (a) Position-velocity plot of the ${ }^{12} \mathrm{CO} J=3-2$ and $\mathrm{H}^{13} \mathrm{CN} J=4-3$ emission from IRAS 16342 along PA $=67^{\circ}$. $X$-axis shows spatial offset from the nebular center (marked by dashed vertical line). $Y$-axis shows velocity offset from the systemic velocity $\left(V_{\mathrm{lsr}}=45 \mathrm{~km} \mathrm{~s}^{-1}\right.$, marked by dashed horizontal lines). The colorscale is in units of Jy beam ${ }^{-1}$; the beam-size (FWHM) is $0.49 \times 0.27$. (b) Spectra of the spatially integrated (total) flux density for ${ }^{12} \mathrm{CO} J=3-2,{ }^{13} \mathrm{CO} J=3-2$ (scaled by factor 1.4), and $\mathrm{H}^{13} \mathrm{CN} J=4-3$ (scaled by a factor of 7 and shifted upward by $1 \mathrm{Jy}$ ). The contribution of emission from the red wing of ${ }^{12} \mathrm{CO} J=3-2$ to that of the blue wing of $\mathrm{H}^{13} \mathrm{CN} J=4-3$ (and vice versa) has been subtracted. Inset shows the mean ${ }^{12} \mathrm{CO}$ and ${ }^{13} \mathrm{CO} J=3-2$ spectra extracted from 0 !. 15 diameter apertures located at $r= \pm 0$.! 6 and the mean $\mathrm{H}^{13} \mathrm{CN} J=4-3$ spectrum extracted from annular wedges with radius (width) \pm 0 !" $6\left(0{ }^{\prime \prime} 12\right)$ and opening angle of $52^{\circ}$, along PA $=133^{\circ}$.

pair are about 110 years old. The corresponding inclination angle ${ }^{9}$ for the $B 1-R 1$ axis is then $8^{\circ}$, and the resulting locations of $B 1$ and $R 1$ are consistent with an extrapolation of the S-shape defined by the other blobs. We conclude that the HVO expansion age is $\sim 110$ years. Note that since the kinematics of the $\mathrm{CO}$ emitting blobs may depend on the hydrodynamic interaction between the underlying jet and surrounding CSE material, the assumption of a uniform radial outflow velocity is likely not strictly true, and the derived ages are somewhat uncertain.

Excluding the velocity range $0<V_{\mathrm{lsr}}\left(\mathrm{km} \mathrm{s}^{-1}\right)<90$ that includes emission from the AGB CSE (see Section 3.4), we find that the velocity-integrated fluxes in the HVO's red wing $\left(90<V_{\mathrm{lsr}}\left(\mathrm{km} \mathrm{s}^{-1}\right)<320\right)$ and blue wing $\left(-228<V_{\mathrm{lsr}}\left(\mathrm{km} \mathrm{s}^{-1}\right)<0\right)$ are 103.9 and $89.8 \mathrm{Jy} \mathrm{km} \mathrm{s}^{-1}$, respectively.

The ${ }^{13} \mathrm{CO}$ to ${ }^{12} \mathrm{CO}$ line intensity ratio is almost unity toward the center, implying a very low relative abundance ratio, $f_{12 / 13}=f\left({ }^{12} \mathrm{CO}\right) / f\left({ }^{13} \mathrm{CO}\right)$. Hence, here and in the following sections, we assume that $f_{12 / 13} \sim 3$, the lowest value achievable via equilibrium $\mathrm{CNO}$ nucleosynthesis, and take $f\left({ }^{12} \mathrm{CO}\right)=3 \times 10^{-4}$, or $f\left({ }^{13} \mathrm{CO}\right)=10^{-4}$. Larger values of $f_{12 / 13}$ will imply proportionately larger values of mass and mass-dependent quantities.

The scalar momentum and kinetic energy in the HVO, derived from the ${ }^{12} \mathrm{CO} J=3-2$ data, assuming the emission to be optically thin (and distance $2 \mathrm{kpc}$ ), is $P_{\mathrm{HVO}}>1.7 \times 10^{38}$ $\mathrm{g} \mathrm{cm} \mathrm{s}^{-1}$ and $E_{\mathrm{HVO}}>3.2 \times 10^{45} \mathrm{erg}$, using the formulation described in Bujarrabal et al. (2001). We adopt an inclination

\footnotetext{
9 All inclination angles, here and elsewhere in the Letter, are relative to the los.
}

angle of $\theta=43^{\circ}$ (see Section 3.2). The HVO mass is $M_{\mathrm{HVO}}=6.2 \times 10^{-3} M_{\odot}$. We assume a uniform excitation temperature, $T_{\text {exc }}=15-30 \mathrm{~K}$ (resulting in a $\sim \pm 20 \%$ uncertainty in $P_{\mathrm{HVO}}, E_{\mathrm{HVO}}$, and $\left.M_{\mathrm{HVO}}\right)$. The $\mathrm{HVO}$ mass-loss rate is $>5.6 \times 10^{-5} M_{\odot} \mathrm{yr}^{-1}$. For the EHVO we find that the mass, scalar momentum $\left(P_{\mathrm{EHVO}}\right)$, and kinetic energy $\left(E_{\mathrm{EHVO}}\right)$ are $7 \%$, $21 \%$, and $51 \%$ of the corresponding values for the HVO; its deprojected speed is $360-540 \mathrm{~km} \mathrm{~s}^{-1}$, and expansion age is 130-305 years, allowing for the EHVO's inclination angle to vary from that of the $\mathrm{HVO}$ by $\sim \pm 12^{\circ}$, similar to the difference in their position angles.

The values of $P_{\mathrm{HVO}+\mathrm{EHVO}}$ and $E_{\mathrm{HVO}+\mathrm{EHVO}}$ in IRAS 16342 lie within the ranges of these quantities for PPNe, $10^{37-40}$ $\mathrm{g} \mathrm{cm} \mathrm{s}^{-1}$ and $10^{42-46}$ erg (Bujarrabal et al. 2001). The HVO cannot be driven by radiation pressure because its dynamical (expansion) timescale is much smaller than that required by radiation pressure to accelerate the observed bipolar outflow to its current speed, $t_{\mathrm{rad}}>P_{\mathrm{HVO}} /(L / c) \sim 7.3 \times 10^{3}$ years, given IRAS 16342's luminosity of $L_{*}=6000 L_{\odot}$.

\subsection{A Central Torus}

The $\mathrm{H}^{13} \mathrm{CN} J=4-3$ emission in channels near the systemic velocity, $V_{\text {lsr }}=43.86-46.4 \mathrm{~km} \mathrm{~s}^{-1}$ (Figure 4(a)), shows two blobs along an axis at $\mathrm{PA} \sim 133^{\circ}$ implying an inclined, equatorial torus or squat-cylindrical structure. The panels at $V_{\text {lsr }}=64.2-61.66 \mathrm{~km} \mathrm{~s}^{-1}\left(V_{\mathrm{lsr}}=31.14-28.6 \mathrm{~km} \mathrm{~s}^{-1}\right)$ presumably show the redshifted (blueshifted) parts of this structure to the SW (NE), consistent with it being an equatorial structure that engirdles the bipolar lobes. A PV plot along the torus major axis (Figure 4(b)) shows the characteristic elliptical shape expected for an expanding torus (since the beam-size has a relatively large extent at $\mathrm{PA} \sim 43^{\circ}$, the cut shows emission 


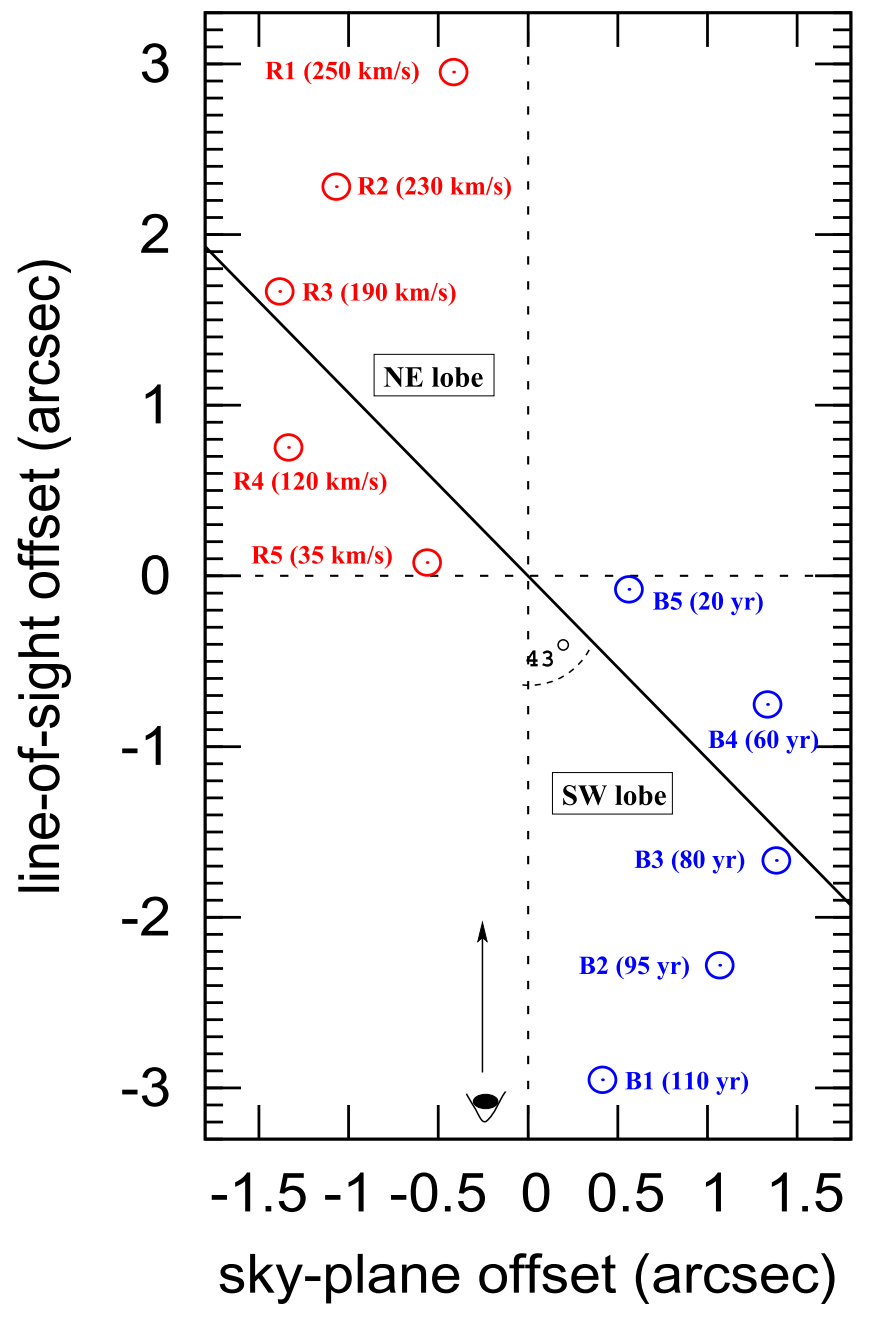

Figure 3. Blob locations in the SW and NE lobes along the line-of-sight (los), derived assuming a uniform outflow velocity $\left(250 \mathrm{~km} \mathrm{~s}^{-1}\right.$ ). Blob ages (radial velocities), which are identical for identically numbered blobs, are shown for the SW (NE) lobe. Since we have assumed that the outflow velocity is the same as that of blobs $B 1$ and $R 1$, we cannot directly derive their los offsets; their adopted locations are based on other considerations (see the text). The sloping black line shows the axis of the central torus.

from all regions of the torus). The observed PV plot along the minor axis (Figure 4(c)) shows the approaching (receding) parts of the torus. Using the tilted torus's projected size along its major axis $(0$ ". 65) and minor axis $(0$ ". 44) as measured from the PV plots, we find that the torus size is $1300 \mathrm{au}$, and its inclination is $43^{\circ}$, consistent with that of the bipolar lobes, $\sim 40^{\circ}$ (Setal99), and the high-velocity $\mathrm{H}_{2} \mathrm{O}$ outflow axis, $\sim 45^{\circ}$ (Claussen et al. 2009). The deprojected torus expansion velocity is $V_{\text {tor }}=20 \mathrm{~km} \mathrm{~s}^{-1}$, and its expansion timescale is 160 years.

We show that the torus density is quite high. The observed peak $\mathrm{H}^{13} \mathrm{CN} J=4-3$ intensity in the torus at the systemic velocity is $37.5 \mathrm{mJy}$ beam $^{-1}\left(T_{b} \sim 8.8 \mathrm{~K}\right)$, implying a minimum $\mathrm{H}^{13} \mathrm{CN}$ column density of $\mathrm{N}\left(\mathrm{H}^{13} \mathrm{CN}\right)=4 \times 10^{15} \mathrm{~cm}^{-2}$ $\left(\delta V / 1 \mathrm{~km} \mathrm{~s}^{-1}\right)$, for optically thin emission and a volume density adequate for exciting the $\mathrm{H}^{13} \mathrm{CN} J=4-3$ line $(\delta V$ is the intrinsic line-width). This result is not sensitive to the assumed excitation temperature, $65 \mathrm{~K}$, derived from the ${ }^{12} \mathrm{CO}$ and ${ }^{13} \mathrm{CO} J=3-2$ line intensities at the torus radius, $r \sim 0$ ". 32 . Taking the radiatively connected column contributing to the line-center emission in the expanding torus to be $\delta z=r \delta V / V_{\text {tor }}$, we find $\delta z=5 \times 10^{14} \mathrm{~cm}\left(\delta V / 1 \mathrm{~km} \mathrm{~s}^{-1}\right)$ at $r=0$ ". 32. If we assume the fractional abundance of $\mathrm{H}^{13} \mathrm{CN}$ to be one-third that of $\mathrm{HCN}, f(\mathrm{HCN})$, then $\mathrm{n}\left(\mathrm{H}_{2}\right)=3 \mathrm{~N}\left(\mathrm{H}^{13} \mathrm{CN}\right) /$ $(f(\mathrm{HCN}) \delta z)=1.1 \times 10^{8} \mathrm{~cm}^{-3}$, taking $f(\mathrm{HCN})=2.2 \times 10^{-7}$ from observations of the oxygen-rich AGB star IK Tau (Decin et al. 2010). If we assume theoretical values, $f(\mathrm{HCN})=9.06 \times 10^{-5} \quad$ (Cherchneff 2006) or $f(\mathrm{HCN})=2.12 \times 10^{-6} \quad$ (Duari et al. 1999), then $n\left(\mathrm{H}_{2}\right)=2.7 \times 10^{5}-1.1 \times 10^{7} \mathrm{~cm}^{-3}$. Assuming that the ratio of the peak torus $\mathrm{H}^{13} \mathrm{CN} J=4-3$ intensity relative to that in the AGB CSE, (say) at $r=0$ ". $75(>14)$ is comparable to the corresponding density ratio, we infer that the torus density is $>3.3 \times 10^{6} \mathrm{~cm}^{-3}$, and $f(\mathrm{HCN})<7.3 \times 10^{-6}$ (the AGB CSE density at $r=0 . \prime 75$ is $\sim 2.4 \times 10^{5} \mathrm{~cm}^{-3}$; see Section 3.4).

\subsection{The Central Continuum Source}

We detect a compact, marginally resolved continuum source at the center, ${ }^{10}$ with (FWHM, undeconvolved) size $0 . " 64 \times 0$. ". 48 , $\mathrm{PA}=80^{\circ}$ (beam is $0.50 \times 0$ ! $26, \mathrm{PA}=-78^{\circ} .2$ ). The peak intensity and integrated flux are $0.25 \mathrm{Jy} \mathrm{beam}^{-1}$ and $0.69 \mathrm{Jy}$ at $\nu=338.19 \mathrm{GHz}$.

The $0.87 \mathrm{~mm}$ continuum flux density observed with the 12m APEX telescope's 18". 6 beam, $602 \pm 90$ mJy (Ladjal et al. $2010)$ is within errors, the same as our observed value (690 mJy). A $1.3 \mathrm{~mm}$ flux density of $277 \pm 13 \mathrm{mJy}$ (Guertler et al. 1996) was measured with the SEST 23" beam. Both these flux densities are significantly in excess of the values $(\sim 113$ and $30 \mathrm{mJy}$ at 0.886 and $1.3 \mathrm{~mm}$ ) derived from an extrapolation of the 2D dust radiative-transfer model fit to IRAS 16342's SED and near-IR polarized light imaging (Figure 1 of Murakawa \& Izumiura 2012), in which the dominant mass component is a $1 M_{\odot}$ torus of radius $700-1000$ au that includes grains with a radius up to $10 \mu \mathrm{m}$.

Assuming that the excess 0.89 and $1.3 \mathrm{~mm}$ flux densities (577 and $247 \mathrm{mJy}$ ) are due to thermal dust emission ${ }^{11}$ from the same region, we find that the dust emissivity power-law $\left(\chi_{\nu} \propto \nu^{\beta}\right)$ index is $\beta \sim 0.46-0.27$ for a dust temperature of, $T_{d} \sim 30-120 \mathrm{~K}$ (e.g., $T_{d} \sim 50 \mathrm{~K}$ for the large grains in the torus of the PPN, IRAS 22036+5306; Sahai et al. 2006). The characteristic dust emission radius, assuming complete absorption of the starlight by intervening dust very close to the star and re-radiation at an effective temperature of $T_{*}=650 \mathrm{~K}$ (Setal05), is given by $r_{d}=\left[\frac{L_{*} T_{*}^{\beta}}{16 \pi \sigma}\right]^{1 / 2} T_{d}^{-(2+\beta / 2)}($ Setal99) and lies in the range $r_{d} \sim(2.0-0.08) \times 10^{17} \mathrm{~cm}$. Since the observed outer radius of the dust emitting source, derived from its major axis (FWHM) of $0 .{ }^{\prime \prime} 64$, is $\sim 0.1 \times 10^{17} \mathrm{~cm}$, we infer that $T_{d} \sim 120 \mathrm{~K}$ and $\beta \sim 0.27$. The low value of $\beta$ at millimeter/ submillimeter wavelengths implies that the grains are very large $(>$ few $\mathrm{mm})$; taking $\chi_{\nu}(0.85 \mathrm{~mm})=1.4 \mathrm{~cm}^{2} \mathrm{~g}^{-1}$ (Sahai et al. 2011), the dust mass of this large-grain component is, $M_{d}=0.017 M_{\odot}$. Testing alternatives to the large-grain model, such as spinning dust and magnetic nanoparticles (e.g., Draine \& Hensley 2012), will require continuum measurements at additional millimeter/submillimeter wavelengths.

\footnotetext{
${ }_{11}^{10}$ Peak located at (J2000) R.A. $=16: 37: 39.935$, decl. $=-38: 20: 17.15$.

11 The contribution of free-free emission is negligible given the upper limit on radio emission by Sahai et al. (2011).
} 

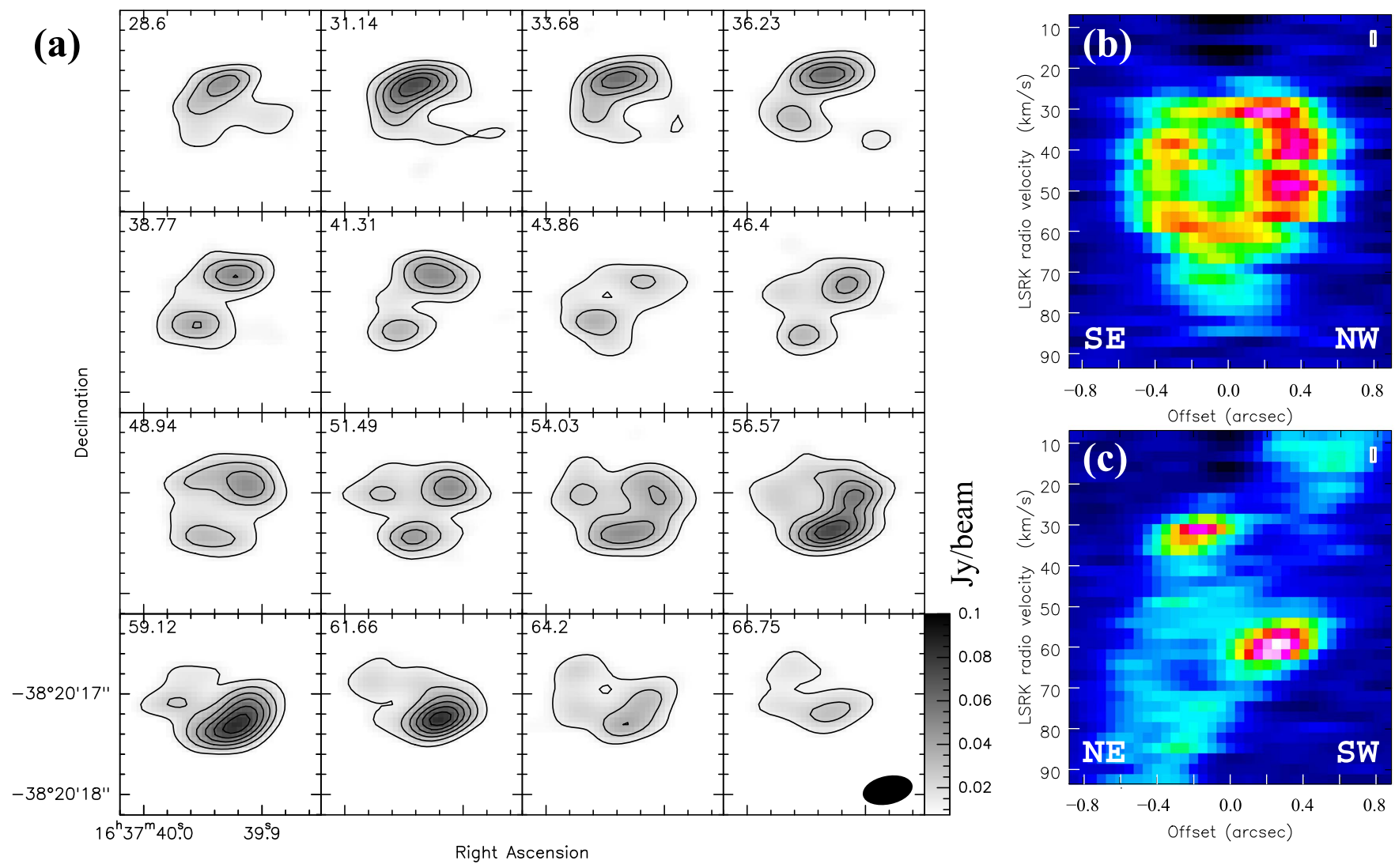

Figure 4. $\mathrm{H}^{13} \mathrm{CN} J=4-3$ emission from IRAS 16342: (a) channel maps near the systemic velocity, showing a tilted torodial structure with major axis along $\mathrm{PA}=133^{\circ}$ (black ellipse in $V_{\mathrm{lsr}}=66.75 \mathrm{~km} \mathrm{~s}^{-1}$ panel shows beam FWHM and orient, and the minimum contour level and step is $0.0125 \mathrm{Jy}$ beam ${ }^{-1}$ ); positionvelocity cuts along the (b) major axis and (c) minor axis of the torus.

\subsection{The Progenitor AGB Circumstellar Envelope}

Our ALMA data provide direct evidence for the jet, and the presence of a surrounding CSE, that are needed for creating the observed bipolar morphology in IRAS 16342 as proposed earlier (Setal99, Setal05). In order to determine the CSE size, we first determine the radial direction along which the ${ }^{12} \mathrm{CO} J=3-2$ line profile is minimally affected by the bipolar outflow from an inspection of the spectra in regions offset from the center in a direction orthogonal to the bipolar nebula. We find that the profile remains centered at the systemic velocity at all positions along $\mathrm{PA} \sim 133^{\circ}$. We then determine the mean ${ }^{12} \mathrm{CO}$ and ${ }^{13} \mathrm{CO}$ intensities at $V_{\mathrm{lsr}}=44 \mathrm{~km} \mathrm{~s}^{-1}$, i.e., the spectral channel closest to the systemic velocity, in two wedges of angular width $26^{\circ}$ oriented along $\mathrm{PA} \sim 133^{\circ}$ and $\mathrm{PA} \sim-47^{\circ}$ as a function of radius, and average these. The resulting intensity cut traces emission from the AGB CSE out to a radius, $R_{\text {out }} \sim 1$." 1 . At $r>R_{\text {out }}$, the intensity falls steeply (Figure 5).

The mean ${ }^{12} \mathrm{CO},{ }^{13} \mathrm{CO}$, and $\mathrm{H}^{13} \mathrm{CN}$ line profiles at $r= \pm 0$ !" 6 along PA $\sim 133^{\circ}$ have widths at their base (FWZI) of $\sim 100,85$, and $38 \mathrm{~km} \mathrm{~s}^{-1}$ (Figure 2(b), inset). It is likely that the optically thick $\mathrm{CO}$ line profiles include contributions from low-density outflow material that does not emit significantly in the $\mathrm{H}^{13} \mathrm{CN}$ $J=4-3$ line. The narrow $\mathrm{H}^{13} \mathrm{CN} J=4-3$ line emission can be seen out to $r \sim 0$ ". 75; we conclude that the FWZI of the latter provides the best estimate of the AGB CSE expansion velocity, i.e., $V_{\mathrm{AGB}} \sim 19 \mathrm{~km} \mathrm{~s}^{-1} /\left[1-\left(0,6 / R_{\mathrm{out}}\right)^{2}\right]^{0.5} \sim 23 \mathrm{~km} \mathrm{~s}^{-1}$.

The radial ${ }^{13} \mathrm{CO}$ and ${ }^{12} \mathrm{CO} J=3-2$ line intensities are very close to each other at all radii $r<\sim 0$ "' 85 , implying that both of these lines are quite optically thick in this region. The

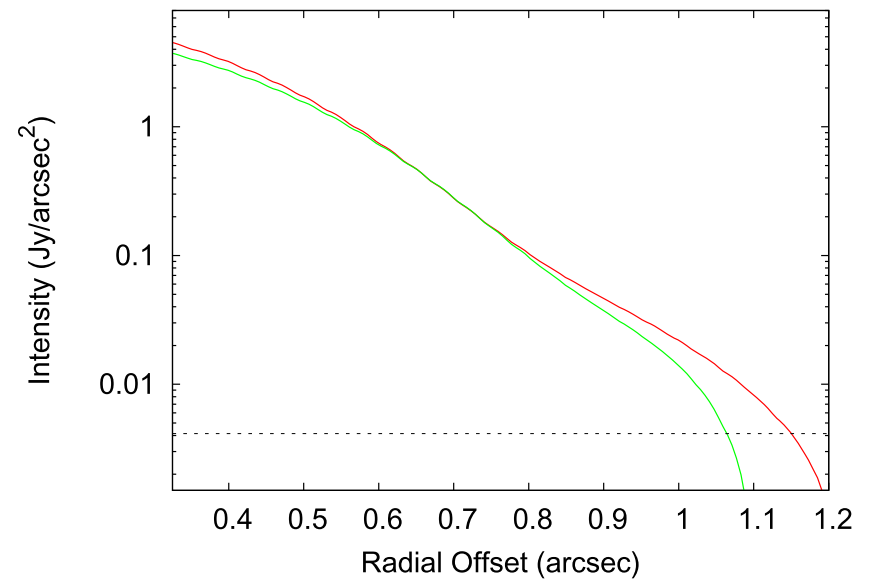

Figure 5. Mean radial ${ }^{12} \mathrm{CO}$ (red) and ${ }^{13} \mathrm{CO}$ (green) $J=3-2$ intensity in the central velocity channel $\left(V_{\mathrm{lsr}}=44 \mathrm{~km} \mathrm{~s}^{-1}\right)$, in wedges of angular width $26^{\circ}$ oriented along $\mathrm{PA}=133^{\circ}$ and $\mathrm{PA}=-47^{\circ}$ (data from the two wedges have been averaged). The dashed horizontal line shows the $1 \sigma$ noise level.

increasing difference between the ${ }^{13} \mathrm{CO}$ and ${ }^{12} \mathrm{CO}$ intensities for $r>\sim 0$ !" 85 signifies a decrease in the optical depth of these lines. From the observed ${ }^{12} \mathrm{CO}$ to ${ }^{13} \mathrm{CO} J=3-2$ intensity ratio of 1.56 at $r=1$, we derive a ${ }^{13} \mathrm{CO} J=3-2$ optical depth of $\tau_{13} \sim 1$, an excitation temperature $T_{\text {exc }} \sim 4.1 \mathrm{~K}$, and a massloss rate $\dot{M}_{\mathrm{AGB}}>3.5 \times 10^{-4}\left(V_{\mathrm{AGB}} / 23 \mathrm{~km} \mathrm{~s}^{-1}\right) M_{\odot} \mathrm{yr}^{-1}$.

At $r>R_{\text {out }}$, both the ${ }^{13} \mathrm{CO}$ and ${ }^{12} \mathrm{CO} J=3-2$ intensities, and their ratio, $R_{I(13 / 12)}$, decrease rapidly-e.g., for a $4 \%$ increase in radius beyond $r=1$ " $0, R_{I(13 / 12)}$ decreases by $28 \%$. This decrease in $R_{I(13 / 12)}$ must result from a more rapid 
decrease in the ${ }^{13} \mathrm{CO}$ optical depth (and thus its density) compared to ${ }^{12} \mathrm{CO}$ and cannot be due to interferometric loss of flux, especially since the maximum recoverable scale is $4^{\prime \prime}$. Without a sharp decrease in density in this region $\left(\sim 10^{5} \mathrm{~cm}^{-3}\right.$ at $\left.r \sim R_{\text {out }}\right), R_{I(13 / 12)}$ could not decrease rapidly because $T_{\text {exc }}$ for both ${ }^{13} \mathrm{CO}$ and ${ }^{12} \mathrm{CO} J=3-2$ would remain very close to $T_{\text {kin }}$, and a sharp decrease in $T_{\text {kin }}$ is unlikely. We therefore conclude that there is preferential photodissociation of ${ }^{13} \mathrm{CO}$ over ${ }^{12} \mathrm{CO}$ by the interstellar UV at $r \gtrsim 1^{\prime \prime}$, implying that the mass-loss rate at $r \gtrsim R_{\text {out }}$ must be $<0.7 \times 10^{-6} M_{\odot} \mathrm{yr}^{-1}\left(V_{\mathrm{AGB}} / 23 \mathrm{~km} \mathrm{~s}^{-1}\right)^{0.5}$ (Mamon et al. 1988). The AGB expansion timescale at $r=R_{\text {out }}$ is 455 years $\left(23 \mathrm{~km} \mathrm{~s}^{-1} / V_{\mathrm{AGB}}\right)$.

\section{Discussion and Concluding Remarks}

Our ALMA study of IRAS 16342 has characterized four different circumstellar components. The oldest of these is the AGB CSE ( 455 years), followed by the EHVO (130-305 years) and torus (160 years), and the youngest is the HVO ( $\sim 110$ years). This sequence suggests that the torus and HVO were formed a few hundred years after a sharp increase in the mass-loss rate of the progenitor AGB star. The HVO (and possibly the EHVO) appears very soon after torus formation in IRAS 16342. Huggins (2007) derives the same result for a small sample of similar objects (late AGB stars, $\mathrm{PPNe}$, and young PNe) and shows that it naturally favors the class of models in which a companion interacts with the central star. These include models where the build-up of a torus enhances the accretion rate in a disk around a companion that then drives jet-like outflows, or both spin-up and ejection of the stellar envelope of the primary occur during a commonenvelope (CE) phase as the companion spirals in to the center of the AGB star. The detailed point-symmetry seen in IRAS 16342's outflows suggests a stable precessing or wobbling disk and argues against explosive scenarios (e.g., Matt et al. 2006).

If the jet activity in IRAS 16342 is driven by an accretion disk around a companion, then we can constrain the type of accretor as done in the study by Blackman \& Lucchini (2014, hereafter BL14), who determine the minimum required accretion rates based on the properties of collimated outflows in PPNe, and compare these with the results of theoretical models. Using Equation (6) of BL14, we scale the accretion rate for IRAS 16342's HVO relative to that of CRL 618 and find that $\dot{M}_{a}\left(\right.$ IRAS 16342) $\sim 0.2 \times \dot{M}_{a}($ CRL 618). Hence, for IRAS 16342 (as for 15/19 objects in BL14's sample), BondiHoyle-Lyttleton wind accretion and wind Roche-lobe overflow (M-WRLOF) accretion are ruled out for both white-dwarf and main-sequence accretors. Enhanced RLOF from the primary (i.e., at the Red Rectangle level; BL14) or accretion within CE evolution appear to be the most likely candidates for powering the HVO in IRAS 16342.

We are grateful to the late Patrick Huggins, who helped in defining the proposal that led to this study. We thank an anonymous referee for helpful comments. R.S.'s contribution to this research was carried out at JPL, California Institute of Technology, under a contract with NASA. W.V., C.S.C., and G.Q.-L. acknowledge support from ERC consolidator grant 614264, Spanish MINECO grant AYA2012-32032, and ERC Grant Agreement 610256 (NANOCOSMOS), respectively. This Letter uses ALMA data set ADS/JAO. ALMA\#2012.1.00678.S. ALMA is a partnership of ESO (representing its member states), NSF (USA) and NINS (Japan), together with NRC (Canada), NSC and ASIAA (Taiwan), and KASI (Republic of Korea), in cooperation with the Republic of Chile. The Joint ALMA Observatory is operated by ESO, AUI/NRAO and NAOJ.

\section{References}

Balick, B., \& Frank, A. 2002, ARA\&A, 40, 439

Balick, B., Huarte-Espinosa, M., Frank, A., et al. 2013, ApJ, 772, 20

Blackman, E. G., \& Lucchini, S. 2014, MNRAS, 440, L16

Bujarrabal, V., Castro-Carrizo, A., Alcolea, J., \& Sánchez Contreras, C. 2001, A\&A, 377, 868

Castro-Carrizo, A., Quintana-Lacaci, G., Neri, R., et al. 2010, A\&A, 523, A59

Cherchneff, I. 2006, A\&A, 456, 1001

Claussen, M. J., Sahai, R., \& Morris, M. R. 2009, ApJ, 691, 219

Cox, P., Lucas, R., Huggins, P. J., et al. 2000, A\&A, 353, L25

Decin, L., De Beck, E., Brünken, S., et al. 2010, A\&A, 516, A69

Draine, B. T., \& Hensley, B. 2012, ApJ, 757, 103

Duari, D., Cherchneff, I., \& Willacy, K. 1999, A\&A, 341, L47

Gledhill, T. M., \& Forde, K. P. 2012, MNRAS, 421, 346

Guertler, J., Koempe, C., \& Henning, T. 1996, A\&A, 305, 878

He, J. H., Imai, H., Hasegawa, T. I., Campbell, S. W., \& Nakashima, J. 2008, A\&A, 488, L21

Huggins, P. J. 2007, ApJ, 663, 342

Imai, H. 2007, in Proc. IAU Symp. 242, Astrophysical Masers and their Environments, ed. J. M. Chapman \& W. A. Baan (Cambridge: Cambridge Univ. Press), 279

Imai, H., Chong, S. N., He, J.-H., et al. 2012, PASJ, 64, 98

Ladjal, D., Justtanont, K., Groenewegen, M. A. T., et al. 2010, A\&A, 513, A53

Lee, C.-F., \& Sahai, R. 2003, ApJ, 586, 319

Likkel, L., \& Morris, M. 1988, ApJ, 329, 914

Likkel, L., Morris, M., \& Maddalena, R. J. 1992, A\&A, 256, 581

Mamon, G. A., Glassgold, A. E., \& Huggins, P. J. 1988, ApJ, 328, 797

Matt, S., Frank, A., \& Blackman, E. G. 2006, ApJL, 647, L45

Murakawa, K., \& Izumiura, H. 2012, A\&A, 544, A58

Neri, R., Kahane, C., Lucas, R., Bujarrabal, V., \& Loup, C. 1998, A\&AS, 130,1

Olofsson, H., Vlemmings, W. H. T., Maercker, M., et al. 2015, A\&A, 576, L15

Sahai, R., Le Mignant, D., Sánchez Contreras, C., Campbell, R. D., \& Chaffee, F. H. 2005, ApJL, 622, L53

Sahai, R., Morris, M. R., \& Villar, G. G. 2011, AJ, 141, 134

Sahai, R., Sánchez Contreras, C., Morris, M., \& Claussen, M. 2007, ApJ, 658,410

Sahai, R., Te Lintel Hekkert, P., Morris, M., Zijlstra, A., \& Likkel, L. 1999, ApJL, 514, L115

Sahai, R., \& Trauger, J. T. 1998, AJ, 116, 1357

Sahai, R., Vlemmings, W. H. T., Huggins, P. J., Nyman, L.-^̊., \& Gonidakis, I. 2013, ApJ, 777, 92

Sahai, R., Young, K., Patel, N. A., Sánchez Contreras, C., \& Morris, M. 2006, ApJ, 653, 124

Siódmiak, N., Meixner, M., Ueta, T., et al. 2008, ApJ, 677, 382

Verhoelst, T., Waters, L. B. F. M., Verhoeff, A., et al. 2009, A\&A, 503, 837 